\title{
Utilización de Eichhornia crassipes y Lemna minor en la remoción de nitrógeno y fósforo de las aguas residuales de la laguna de oxidación de la ciudad de Pucallpa, Perú
}

\author{
Katia Lisbeth Quispe Benavides \\ katia.qb89@gmail.com \\ Consultora ambiental de CCIMA SAC \\ Pucallpa - Perú \\ Noe Klever Guadalupe Baylón \\ iagubanok@gmail.com \\ Universidad Nacional de Ucayali \\ Pucallpa - Perú \\ Hugo Diaz Avalos \\ hugodz92@gmail.com \\ Gobierno Regional de Ucayali \\ Pucallpa - Perú \\ Hugo Guillermo Diaz Panduro \\ dphugocpc@hotmail.com \\ Universidad Nacional de Ucayali \\ Pucallpa - Perú
}

\section{RESUMEN}

El presente estudio tuvo como objetivo determinar la capacidad de remoción de nitrógeno (N) y fósforo (P) en Aguas Residuales Municipales (ARM), por plantas acuáticas emergentes. Se utilizaron plantas nativas de la región en los efluentes de la Planta de Recuperación de la Empresa Municipal de Agua Potable y Alcantarillado - EMAPACOP S.A. (Pucallpa, Perú), aplicando dos métodos de investigación experimental y aplicada; se evaluó la capacidad de remoción de $\mathrm{N}$ y $\mathrm{P}$ durante 5 días, a nivel laboratorio, en ARM. La Eichhornia crassipes y Lemna minor, tuvieron un buen crecimiento en ARM. El cultivo de plantas acuáticas emergentes, se realizó con 4 meses de crecimiento en condiciones controladas de luz, temperatura y $\mathrm{pH}$. El cultivo de Eichhornia crassipes y Lemna minor, reportó valores altos de remoción, entre 70\%-80\%, y 55\%-60\%, respectivamente. La cantidad óptima de plantas acuáticas emergentes, para tratar aguas residuales y disminuir el exceso de nutrientes causantes de eutrofización o hipoxia, fue de 60 muestras para tratar 80 litros de agua cruda, con una disminución de 0,35 mg/l hasta $0,09 \mathrm{mg} / \mathrm{l}$ de nitrógeno y de $5 \mathrm{mg} / \mathrm{l}$ hasta $0,53 \mathrm{mg} / \mathrm{l}$ de fósforo.

Palabras clave: Aguas residuales; eutrofización; especies acuáticas; cantidad óptima; remoción 


\title{
Use of Eichhornia crassipes and Lemna minor in the removal of nitrogen and phosphorus from the wastewater of the oxidation lagoon of the city of Pucallpa, Perú
}

\begin{abstract}
The objective of this study was to determine the nitrogen $(\mathrm{N})$ and phosphorus $(\mathrm{P})$ removal capacity in Municipal Wastewaters (ARM), by emerging aquatic plants. Native plants of the Region from the tributaries of the Recovery Plant of the Drinking Water and Sewer Municipal Company - EMAPACOP S.A (Pucallpa, Perú) were used, applying two research methods, experimental and applied; its $\mathrm{N}$ and $\mathrm{P}$ removal capacity was evaluated during 5 days, at laboratory level, in ARM. The Eichhornia crassipes and Lemna minor, had a good growth in ARM. The cultivation of emergent aquatic plants was carried out with 4 months of growth under controlled conditions of light, temperature and $\mathrm{pH}$. The cultivation of Eichhornia crassipes and Lemna minor, reported high values of removal, between $70 \%-80 \%$, and 55\%-60\%, respectively. The optimal amount of emergent aquatic plants, to treat wastewater and reduce excess nutrients that cause eutrophication or hypoxia, was 60 samples to treat 80 liters of raw water, with a decrease from $0.35 \mathrm{mg} / 1$ to $0.09 \mathrm{mg} / 1$ of nitrogen and from $5 \mathrm{mg} / 1$ to $0.53 \mathrm{mg} / 1$ of phosphorus.
\end{abstract}

Keywords: Wastewater, eutrophication, aquatic species, optimal quantity, removal.

Artículo recibido: 05 de Abril 2021 Aceptado para publicación: 28 de Mayo 2021

Correspondencia: hugodz92@gmail.com Conflictos de Interés: Ninguna que declarar 


\section{INTRODUCCIÓN}

El tratamiento de los efluentes domésticos es una necesidad que tiene la sociedad para proteger su medio ambiente y garantizar el bienestar humano, pues éstas configuran un peligro potencial para la salud pública, ya que a través de las mismas se pueden transmitir innumerables enfermedades; lo cual genera impactos negativos a la población y la economía de los países.

La degradación ambiental causada por los efluentes domésticos, es hoy en día uno de los aspectos de mayor atención. El efluente doméstico puede contener una variedad de exceso de nutrientes que podrían causar impactos negativos al ambiente cuando son liberados.

El exceso de nutrientes, es el principal causante del deterioro de la calidad del agua, generando eutrofización en cuerpos receptores de agua natural. Los nutrientes en los efluentes domésticos provienen de los fertilizantes, baños, entre otros. En su gran mayoría el fósforo viene de los detergentes y el nitrógeno a través de la urea de las heces a la orina. Uno de los mayores problemas del tratamiento de efluentes en el Perú es que no contempla el exceso de nutrientes en la salida de plantas de tratamientos de aguas residuales. El agua que sale de las plantas de tratamientos va hacia un cuerpo natural, propagando alteraciones físicas y químicas de agua.

Este estudio se realizó en el laboratorio de Ciencias de la Salud de la Universidad Nacional de Ucayali, el efluente a tratar se recolecto de la planta de tratamiento de oxidación de la Empresa Municipal de Agua y Alcantarillado de Coronel Portillo del sector 14.

Cabe mencionar que se trabajó con las especies acuáticas emergentes, Eichhornia crassipes y Lemna minor, para saber cuál de especie remueve más rápido el exceso de nutrientes. El objetivo principal de este estudio fue determinar la utilización de estas macrofitas en la remoción de nitrógeno y fósforo, de las aguas residuales de la laguna de oxidación de la Empresa EMAPACOP S.A. en Ucayali.

\section{MATERIALES Y MÉTODOS}

\subsection{Lugar de ejecución}

El trabajo de investigación se realizó en el Laboratorio de Microbiología y Parasitología, perteneciente a la Universidad Nacional de Ucayali, a la Facultad de Ciencias de Salud, en el distrito de Callería, provincia de Coronel Portillo, departamento de Ucayali. 
Las aguas residuales utilizadas en el presente estudio, tanto para la remoción del exceso de nutrientes, fueron obtenidas de la Planta de Tratamiento de Aguas Residuales, de la Empresa Municipal de Agua Potable y Alcantarillado S.A, ubicada en el sector catorce del distrito de Manantay. Esta planta recibe las aguas contaminadas del sector nueve del Distrito de Manantay para someterlas a un pre-tratamiento a base de lagunas de oxidación, es un proceso natural de autodepuración, que incluye un proceso físico de remoción de materia suspendida (Sedimentación) y un proceso químico que mantiene las condiciones adecuadas para que los organismos puedan realizar la estabilización, transformación, y remoción de contaminantes orgánicos biodegradables (Emapacopsa, 2015).

Así mismo las especies Jacinto de agua (Eichhornia crassipes) y lenteja de agua (Lemna minor) se obtuvieron del Parque Natural de Pucallpa.

\subsection{Método de investigación}

En el presente estudio se utilizaron 2 métodos de investigación: Experimental y evaluación.

- Experimental: Debido a que en la presente investigación determinó cuáles de las dos especies de plantas acuáticas remueve más rápido el exceso de nutrientes (nitrógeno y fósforo) en condiciones controladas (Coral, 2002).

- Evaluación: Debido a que los resultados obtenidos fueron evaluados para solucionar el problema del exceso de nutrientes que causa la eutrofización en cuerpos receptores en cuerpos naturales, utilizando plantas acuáticas, así mismo estas plantas acuáticas tienen un tiempo de absorción, a través de su metabolismo pueden asimilar nitrógeno y fósforo (Coral, 2002).

\subsection{Población y muestra}

\section{Población}

Para la ejecución de este estudio se tomó los afluentes de la planta de tratamiento de aguas residuales del sector nueve del distrito de Manantay, cuya ubicación es a 8²5'49' de Latitud y 74³4'29' de Longitud; esta planta funciona bajo la tecnología de lagunas de oxidación, administrado por la Empresa Municipal de Agua Potable y Alcantarillado S.A, la cual posee 4 lagunas primarias de 120 metros de largo x 50 metros de ancho y 2.80 metros de profundidad por cada laguna, con una velocidad de entrada de afluente de 10 1/s, con una $1500 \mathrm{~m} 3$ de volumen al día y 4 segundarias, 90 de largo x 30 metros de ancho 
y 1.5 metros de profundidad por cada una con una velocidad de salida del efluente de 5 1/s (Emapacopsa, 2015).

\section{Muestra}

Los efluentes muestreados fueron tomados de las salidas de las lagunas 1 y 2, el día 10 de octubre del 2018 a las 11:00 am, debido a que las lagunas 3-4 son utilizadas para el tratamiento de aguas residuales de derivados de petróleo. Los análisis se realizaron consecutivamente una vez inmovilizado y sembrado las plantas acuáticas en los estanques de vidrio en el laboratorio a condiciones normales de temperatura, $\mathrm{pH}$ y oxígeno, para saber la disminución del exceso de nutrientes en un día por un periodo de 10 días.

Tabla 1. Puntos de Muestreo

\begin{tabular}{|l|c|}
\hline Puntos de muestreo & Codificación \\
\hline Efluente de la laguna primaria 1 de oxidación & PM01 \\
Efluente de la laguna primaria 2 de oxidación & PM02 \\
\hline
\end{tabular}

\subsection{Procedimientos de recolección de datos}

\section{Sistemas de tratamiento}

Para el desarrollo de esta investigación se utilizó tres tratamientos de flujo discontinuo o también llamado por tandas; que constó de un estanque para cada sistema, el cual simuló a una laguna pequeña con agua estancada.

Los sistemas se realizaron por triplicado con tres repeticiones de 10 días, con las siguientes cantidades de plantas; el primer sistema fue de 20, 40 y 60 plantas de Lenteja de agua (Lemna minor), el segundo sistema fue de 20, 40 y 60 plantas de Jacinto de agua (Eichhornia crassipes) y el tercer sistema fue un estanque sin planta acuática al cual se le llamó control como se muestra en la Tabla 2.

Tabla 2. Tratamientos aplicados en el proceso de aguas residuales.

\begin{tabular}{|c|l|l|l|}
\hline \multicolumn{2}{|c|}{$\mathbf{N}^{\circ}$ de Tratamiento } & $\begin{array}{c}\text { Componentes } \\
\text { (Especies) }\end{array}$ \\
\hline $\mathrm{T} 1=20$ & $\mathrm{~T} 2=40$ & $\mathrm{~T} 3=60$ & Estanque con Eichhornia crassipes \\
\hline $\mathrm{T} 1=20$ & $\mathrm{~T} 2=40$ & $\mathrm{~T} 3=60$ & Estanque con Lemna minor \\
\hline $\mathrm{T}_{1}$ & $\mathrm{~T}_{2}$ & $\mathrm{~T}_{3}$ & Estanque sin plantas acuática flotantes \\
\hline
\end{tabular}


Los estanques con Eichhornia crassipes y Lemna minor se utilizaron como tratamiento secundario debido a que el agua residual primeramente fue tratada en un filtro de grava ya que contenían residuos orgánicos, bolsas y papeles.

\section{Dimensionamiento de los sistemas}

En el estudio de Coral (2002) el diseño de los sistemas que se establece cómo la relación (largo: ancho) de 10:1 para que el flujo del agua residual cumpliera la teoría del flujo pistón. Sin embargo, cuando el sistema de tratamiento es a nivel piloto, García (2012) estableció una relación (largo: ancho) de 2,7; para que el flujo se aproxime a un pistón. Para determinar las dimensiones de los estanques, se estimó un volumen de 80 litros ( 0,08 $\mathrm{m} 3$ ), altura de $30 \mathrm{~cm}$ y un tiempo de retención hidráulica (TRH) de 10 días.

\section{Construcción de los estanques}

Se construyeron 9 estanques de material de vidrio, para el tratamiento de las aguas residuales, con dimensiones $(31,5 \mathrm{~cm}$ de ancho, 85,05 $\mathrm{cm}$ de largo y $30 \mathrm{~cm}$ de profundidad) y con una capacidad de almacenamiento de 80 litros.

\section{Acondicionamiento del lugar}

El espacio que se acondicionó para la instalación de los sistemas de tratamiento y el desarrollo de esta investigación, se ubicó en el Laboratorio de Microbiología y Parasitología de la Facultad de Ciencias de la Salud de la Universidad Nacional de Ucayali.

\section{Recolección de las especies de plantas acuáticas}

Las plantas de Eichhornia crassipes, y Lemna minor fueron recolectados de la planta de tratamiento de aguas residuales de la Empresa Municipal de Agua Potable y Alcantarillado de Coronel Portillo, ubicada en el sector 14, a una temperatura promedio anual de $26{ }^{\circ} \mathrm{C}$.

Las plantas recolectadas fueron sometidas a un período de aclimatación, para evitar que su crecimiento se vea afectado al momento de ser trasplantadas al sistema piloto, por lo cual se destinó un tiempo de 5 días. El intervalo de tiempo propuesto para la aclimatación de las plantas se estimó tomando en consideración la investigación de (Leal, 2006).

\section{Instalación de los sistemas}

Se utilizó nueve (09) estanques de vidrio de las siguientes dimensiones: $30 \mathrm{~cm}$ de profundidad, $31,5 \mathrm{~cm}$ de ancho y 85,05 $\mathrm{cm}$ de largo. El volumen de los estanques fue de 80 litros $(0,08 \mathrm{~m} 3)$. Los estanques fueron alimentados del agua residual de la planta de 
tratamiento de aguas residuales del sector 14 , hasta el $85 \%$ de su altura haciendo un volumen de 76,5 litros $(0,0765 \mathrm{~m} 3)$, el cual fue previamente filtrado en un filtro de grava para quitar los residuos sólidos orgánicos, bolsas y papeles que contenía el agua.

Para la selección de las macrofitas, se utilizó el método de (Lucero, 2009) que consistió en seleccionar los hijuelos de plantas de Eichhornia crassipes y las plantas más jóvenes con un color más verde de Lemna minor.

En la siembra de las macrofitas, se utilizó el método utilizado por García (2012), que consistió en lavar las macrofitas con agua corriente y colocarlos en los estanques hasta cubrir la mitad del área.

\section{Instrumentos de recolección de datos}

Materiales, herramientas y equipos de gabinete:

- Equipos: Laptop HP Core i5, Calculadora y USB 32GB

- Materiales: Lapicero, libreta, botellas de plástico, botellas de vidrio, grava, estanques de vidrio, marcador, cinta masking tape y papel toalla

- Herramientas: Machete y baldes de 80 litros

- Reactivos: Agua desionizada y alcohol etílico $90^{\circ}$

Materiales, herramientas y equipos de campo

- Herramientas: Microsoft 2013, ArcGis 10.3, Autocad 2014, pico, aplanador de concreto, nivel, balde y tina de plástico, cinta métrica, peceras de vidrio de capacidad de 80 litros, fluorescentes (Philips) de 30 watts, marcador, cinta masking tape

- Equipos: Navegador GPS, cámara fotográfica, multiparámetro de nitrógeno y fósforo, turbidimetro, botellas Winkler, probetas, pisetas, pipetas, tubos de vidrio, gradillas, cronometro, celdas, filtro de membrana de vidrio, espectrofotómetro, balanza analítica, equipo de filtración, equipo de titulación graduado y estufa

- Insumos: Plantas acuáticas Eichhornia crassipes y Lemna minor

\section{Toma de muestra}

Según García (2012), recomienda tomar las muestras en las horas donde hay cambio de radiación y por lo tanto variación de la actividad fotosintética en el agua a tratar, todo esto involucra el grado de tratamiento en horas críticas.

Para determinar el horario de muestreo se tomaron las muestras y se realizó el análisis de DQO en las siguientes horas: 9:00, 12:00 y 16:00 horas; ya que según Madueño y 
Sandoval (2009) se debe elegir la hora donde existe un mayor valor de DQO, y en este caso fue a las 9 de la mañana.

Figura 1. Toma de muestra del agua residual de la laguna de oxidación para el análisis en el laboratorio

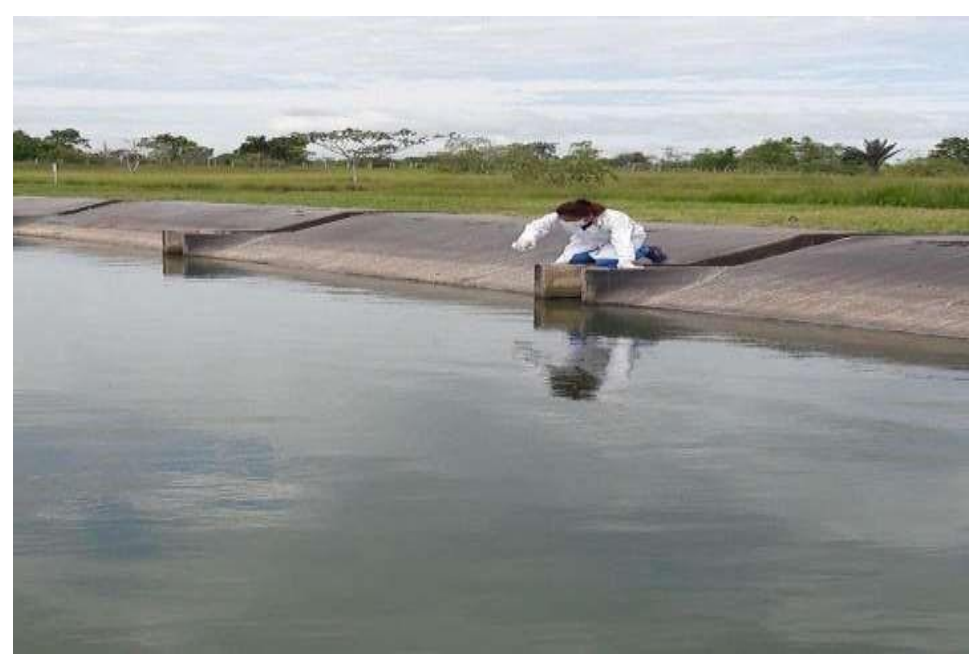

La temperatura y $\mathrm{pH}$ de las muestras se tomaron de forma inmediata. Se colocaron $50 \mathrm{ml}$ de aguas residuales a $4{ }^{\circ} \mathrm{C}$ para su posterior análisis de parámetros fisicoquímicos.

Se tomó por triplicado, 240 litros de aguas residuales domésticas sometidas a pretratamiento de la salida de las lagunas de oxidación 1 y 2, del Sector Nueve del distrito de Manantay. Las muestras se pusieron en frascos de vidrio transparentes utilizados para su traslado al Laboratorio de Microbiología y Parasitología de la Universidad Nacional de Ucayali del distrito de Manantay, en donde se dejó en estantes de vidrio con fluorescentes (Phillips) de 30 watts cada uno, a una distancia de $30 \mathrm{~cm}$ y a temperatura ambiente. Dicha iluminación continua se mantuvo durante los 10 días que duró la fase de remoción.

El efluente estancado fue cambiado cada 10 días según el tiempo de retención hidráulica elegido para esta investigación; el periodo de muestreo fue cada primer día que se alimentó con agua residual los estanques (una muestra del efluente), luego se tomaron muestras diferentes para cada estanque al décimo día.

\section{Análisis de las muestras}

Los análisis para determinar la eficiencia y el comportamiento de los sistemas fueron realizados en el laboratorio.

- Procedimiento en Campo: Se utilizó un equipo multiparámetro para medir pH, conductividad, oxígeno disuelto y temperatura; además de un turbidímetro para la 
medición de turbidez. Los equipos que se utilizaron para la medición de estos parámetros son propiedad del Laboratorio de Microbiología y Parasitología de la Facultad de Ciencias de la Salud, de la Universidad Nacional de Ucayali.

- Procedimiento en laboratorio: Los análisis de los parámetros químicos como Nitrógeno y Fósforo, además de los parámetros microbiológicos se realizaron en el Laboratorio de Microbiología y Parasitología de la Facultad de Ciencias de la Salud, de la Universidad Nacional de Ucayali.

- Tiempo de crecimiento: El tiempo de crecimiento de las plantas fue de 4 meses y se determinó mediante la biomasa (peso fresco y peso seco) se tomaron al inicio del ensayo tres muestras frescas de $5 \mathrm{~g}$ cada una; se llevaron a estufa por 24 horas a una temperatura de $85{ }^{\circ} \mathrm{C}$ para calcular el peso seco. Al final del ensayo se recolectó la totalidad de la biomasa en cada réplica de cada estanque y se realizó el procedimiento nuevamente, para determinar la ganancia de biomasa como peso fresco, el peso seco y el porcentaje de peso seco. Así mismo esta herramienta que permitió saber que tanto crecen las plantas de acuerdo a la biomasa.

\section{Tratamientos de los datos}

Para el análisis estadístico se utilizó el programa SPSS versión 22, mediante el cual se determinaron las diferencias significativas entre los tratamientos realizados a través del programa Anova en la etapa de remoción de nitrógeno y fósforo a escala de laboratorio al comparar los valores.

\section{RESULTADOS Y DISCUSIÓN}

\section{Crecimiento poblacional de plantas acuáticas Eichhornia crassipes y Lemna minor}

El crecimiento de las plantas acuáticas emergentes Eichhornia crassipes y Lemna minor dio como resultado que para los estanques número uno, para ambas especies la cantidad de muestras óptimas para crecimiento fue 20 muestras de plantas, debido a que las especies necesitan un mayor ambiente para su crecimiento a diferencia de las demás peceras dos y tres que también crecieron aunque no en las mismas magnitudes que la primera, esto se debe que a mayor cantidad de muestra se necesita un espacio mayor a un estanque de 80 litros de capacidad. Se tuvo un $\mathrm{pH} 7.01$ para Lemna minor y $7.56 \mathrm{de} \mathrm{pH}$ de Eichhornia crassipes y un crecimiento de $3.1 \mathrm{~cm}$ de raíz para Lemna minor y $18 \mathrm{~cm}$ para Eichhornia crassipes. 
Tabla 3. Crecimiento de plantas acuáticas Eichhornia crassipes y Lemna minor.

\begin{tabular}{|c|c|c|c|c|c|}
\hline \multirow{7}{*}{$\begin{array}{l}\text { Sistemas de } \\
\text { tratamientos - } \\
\text { Longitud de } \\
\text { raíces }\end{array}$} & Plantas & 1 mes & $\begin{array}{c}2 \\
\text { meses }\end{array}$ & $\begin{array}{c}3 \\
\text { meses }\end{array}$ & $\begin{array}{c}4 \\
\text { meses }\end{array}$ \\
\hline & Estanque 1 Lemna minor 20 plantas & $1 \mathrm{~cm}$ & $1.3 \mathrm{~cm}$ & $2.4 \mathrm{~cm}$ & $3.1 \mathrm{~cm}$ \\
\hline & Estanque 2 Lemna minor 40 plantas & $1.3 \mathrm{~cm}$ & $1.8 \mathrm{~cm}$ & $2.2 \mathrm{~cm}$ & $3 \mathrm{~cm}$ \\
\hline & Estanque 3 Lemna minor 60 plantas & $0.8 \mathrm{~cm}$ & $1 \mathrm{~cm}$ & $1.7 \mathrm{~cm}$ & $2.5 \mathrm{~cm}$ \\
\hline & $\begin{array}{l}\text { Estanque } 1 \text { Eichhornia crassipes } \\
20 \text { plantas }\end{array}$ & $5 \mathrm{~cm}$ & $9 \mathrm{~cm}$ & $16 \mathrm{~cm}$ & $18 \mathrm{~cm}$ \\
\hline & $\begin{array}{l}\text { Estanque } 2 \text { Eichhornia crassipes } \\
40 \text { plantas }\end{array}$ & $5 \mathrm{~cm}$ & $7 \mathrm{~cm}$ & $14 \mathrm{~cm}$ & $16 \mathrm{~cm}$ \\
\hline & $\begin{array}{l}\text { Estanque } 3 \text { Eichhornia crassipes } \\
60 \text { plantas }\end{array}$ & $3 \mathrm{~cm}$ & $5 \mathrm{~cm}$ & $9 \mathrm{~cm}$ & $13 \mathrm{~cm}$ \\
\hline
\end{tabular}

Promedio de los resultados de los parámetros analizados en el laboratorio

Tabla 4. Promedio de los resultados por sistemas de estanques.

\begin{tabular}{|c|c|c|c|c|c|c|c|c|}
\hline \multirow{9}{*}{ 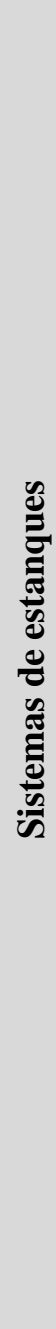 } & ڤ્⿹ & 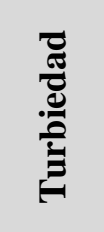 & $\overline{2}$ & 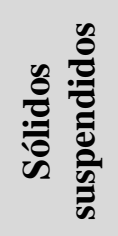 & 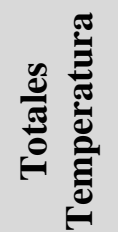 & 赵胥 & 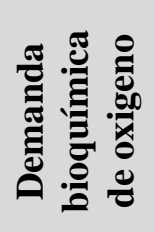 & 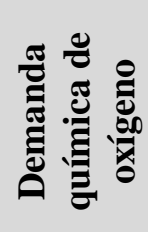 \\
\hline & Unidades & UNT & $\begin{array}{c}\text { Potencial } \\
\text { de } \\
\text { hidrógeno }\end{array}$ & $\mathrm{mg} / \mathrm{l}$ & ${ }^{\circ} \mathrm{C}$ & $\mathrm{mg} / \mathrm{l}$ & $\mathrm{mg} / \mathrm{l}$ & $\mathrm{mg} / \mathrm{l}$ \\
\hline & $\begin{array}{l}\text { Datos } \\
\text { iniciales }\end{array}$ & 80 & 6.64 & 110 & 23 & 2.2 & 168 & 350 \\
\hline & $\begin{array}{l}\text { Estanque } 1 \\
\text { Lemna minor } \\
20 \text { plantas }\end{array}$ & 52 & 5.99 & 91.75 & 22.55 & 6.75 & 138.25 & 339 \\
\hline & $\begin{array}{l}\text { Estanque } 2 \\
\text { Lemna minor } \\
40 \text { plantas }\end{array}$ & 34.5 & 6.73 & 69 & 22.81 & 4.13 & 113.32 & 264.25 \\
\hline & $\begin{array}{l}\text { Estanque } 3 \\
\text { Lemna minor } \\
60 \text { plantas }\end{array}$ & 26.25 & 7.01 & 41.23 & 22.6 & 3.13 & 72.25 & 190.75 \\
\hline & $\begin{array}{l}\text { Estanque } 1 \\
\text { E. crassipes } \\
20 \text { plantas }\end{array}$ & 22.34 & 6.93 & 54.32 & 23.2 & 5.64 & 150.54 & 249.25 \\
\hline & $\begin{array}{l}\text { Estanque } 2 \\
\text { E. crassipes } \\
40 \text { plantas }\end{array}$ & 18.21 & 7.13 & 32.33 & 22.3 & 4.56 & 134.67 & 215.32 \\
\hline & $\begin{array}{l}\text { Estanque } 3 \\
\text { E. crassipes } \\
60 \text { plantas }\end{array}$ & 13.26 & 7.56 & 25.13 & 23.1 & 2.34 & 65.34 & 89.65 \\
\hline
\end{tabular}


Los parámetros analizados en el laboratorio nos dan como resultado que los estanques número tres tanto para Eichhornia crassipes y Lemna minor, son buenos pero el más efectivo entre las dos especies es la Eichhornia crassipes con mejores resultados, prometedores para remediar aguas contaminadas debido a que los sólidos suspendidos totales disminuyeron hasta $25.13 \mathrm{mg} / \mathrm{l}$ y la turbiedad hasta 13.26 UNT como se observa en la tabla 4.

\section{Promedio de los resultados de los parámetros de remoción de nitrógeno y fósforo}

Tabla 5. Parámetros de remoción de nitrógeno y fósforo

\begin{tabular}{|c|l|c|c|}
\hline \multicolumn{1}{|c|}{ Parámetros } & \multicolumn{2}{|c|}{ Nitrógeno } & Fósforo \\
\cline { 2 - 4 } & Días & $\mathrm{mg} / \mathrm{l}$ & $\mathrm{mg} / 1$ \\
\cline { 2 - 4 } & Unidades & 0.35 & 5 \\
\cline { 2 - 4 } $\begin{array}{c}\text { Sistemas de } \\
\text { Estanques }\end{array}$ & $\begin{array}{l}\text { Datos iniciales de nitrógeno y fósforo } \\
\text { Estanques 1 Lemna minor }\end{array}$ & 0.28 & 4.6 \\
\cline { 2 - 4 } & $\begin{array}{l}\text { Estanques 2 Lemna minor } \\
40 \text { plantas }\end{array}$ & 0.18 & 3.2 \\
\cline { 2 - 4 } & $\begin{array}{l}\text { Estanques 3 Lemna minor } \\
60 \text { plantas }\end{array}$ & 0.11 & 1.5 \\
\cline { 2 - 4 } & $\begin{array}{l}\text { Estanques 1 Eichhornia crassipes } \\
\text { 20 plantas }\end{array}$ & 0.25 & 3.56 \\
\cline { 2 - 4 } & $\begin{array}{l}\text { Estanques 2 Eichhornia crassipes } \\
40 \text { plantas }\end{array}$ & 0.15 & 2.12 \\
\cline { 2 - 4 } & $\begin{array}{l}\text { Estanques 3 Eichhornia crassipes } \\
60 \text { plantas }\end{array}$ & 0.09 & 0.53 \\
\hline
\end{tabular}

En la tabla 5 se observa cómo comenzaron los análisis de nitrógeno y fósforo con 0.35 $\mathrm{mg} / \mathrm{l}$ y $5 \mathrm{mg} / \mathrm{l}$ respectivamente, para lo cual las peceras número tres de ambas especies tuvieron buenos resultados, pero la Eichhornia crassipes fue la especie que removió más el exceso de nutrientes causantes de la eutrofización en cuerpos de aguas naturales en el mismo tiempo, el Nitrógeno llegó a un valor de 0.09 mg/l y el Fósforo a 0.53 mg/l.

\section{Discusión}

El crecimiento de las plantas acuáticas Lemna minor y Eichhornia crassipes, tiene un periodo de aclimatación de 5 días, con un periodo de crecimiento de 4 meses de acuerdo al estudio en peceras de tandas, el cual no es continuo si no se cambia el agua cada diez días. Así mismo Valderrama, Campos y Zapata (2002), en su investigación de Lemna minor y Eichhornia crassipes para tratar aguas residuales domésticas, tuvieron un periodo de crecimiento de 2 meses, a diferencia de Cevallos (2015) quien en su estudio utilizó 
plantas acuáticas para remediar aguas residuales pesqueras utilizando Lemna minor y Salvinia, con un periodo de crecimiento de 1 mes y medio. Además, Castro (2006), en su investigación en el tratamiento de aguas residuales de la Universidad Nacional Toribio Rodríguez Mendoza de Amazonas, utilizó el Jacinto de agua y lenteja de agua con un periodo de crecimiento de 1 mes.

En la remoción del exceso de nutrientes causante de la eutrofización de nitrógeno y fósforo en el estudio hubo una disminución para Lemna minor de 55\% - 60\% y para Eichhornia crassipes fue de 70\%-80\%, Valderrama y Campos (2002) registran una disminución de $60 \%$ de nitrógeno y $90 \%$ para fósforo, pero Cevallos (2015), en su estudio de parámetros para el tratamiento de aguas residuales el nitrógeno disminuyó en un 78\%, por otro lado Panduro (2008), en su investigación denominada "Sistema de descontaminación de las aguas residuales mediante el uso de plantas acuáticas, en la provincia de Coronel Portillo, región Ucayali, utilizó Eichhornia crassipes determinando una reducción de nitrógeno y fósforo en $78 \%$ y $80 \%$ en la depuración del exceso de nutrientes.

\section{CONCLUSIONES}

- La remoción de nitrógeno y fósforo de Eichhornia crassipes y Lemna minor fue de $70 \%-80 \%$ y $55 \%-60 \%$ respectivamente.

- El tiempo de crecimiento de las plantas acuáticas en condiciones controladas fue de 4 meses, Lemna minor creció un total de 2,55 cm y Eichhornia crassipes $13 \mathrm{~cm}$ con un periodo de aclimatación de 5 días para ambas especies.

- El tiempo de remoción en condiciones controladas fue de 5 días y la cantidad óptima fue de 60 muestras para ambas especies. Para la especie Lemna minor el nitrógeno comenzó con 0,35 mg/l y disminuyó hasta $0,11 \mathrm{mg} / \mathrm{l}$, para el fósforo comenzó con 5 mg/l y bajó a 0,11mg/l. Para la especie Eichhornia crassipes el nitrógeno comenzó con $0,35 \mathrm{mg} / \mathrm{l}$ y disminuyó hasta $0,09 \mathrm{mg} / \mathrm{l}$ y el fósforo comenzó con $5 \mathrm{mg} / \mathrm{l}$ y descendió hasta $0,53 \mathrm{mg} / \mathrm{l}$.

\section{LISTA DE REFERENCIAS}

Aguilar, N. (2012). Determinación de parámetros fisicoquímicos y microbiológicos para agua apta para el consumo humano de Concepción Quezaltepeque, Chalatenango. 36. San Salvador, El Salvador: Universidad de El Salvador.

Barsanti, G. (2006). Factores que influyen en el crecemiento de las plantas acuáticas. 
Biswas, A. (2010). Cambiar el paisaje global de la gestión del agua. 12-15.

Bitton. (2005). Agua Residual Doméstica.

British Columbia Ministry of Environment. (2003). Ambient Water Quality Guidelines for Chloride. Lands and Parks (BC MELP). 125-141.

Brix, H. (1994). Use of constructed wetlands in water pollution control: Historical development, present status, andfuture perspectives. 209-223. Water Science and Technology.

Caballero, Y. (2007). Potencial hidrobiológico y calidad de las aguas superficiales en la subcuenca del río Ochomogo. 42. Managua, Nicaragua: Universidad Nacional Autónoma de Nicaragua.

Camacho, J., \& Ordoñez, L. (2008). Evaluación de la eficiencia de un sistema de recuperación de aguas residuales con Eichhornia crassipes para el postratamiento del efluente del reactor anaerobio a flujo pistón de la Universidad Pontificia Bolivariana de Bucaramanga. 27- 40. Bucaramanga, Colombia: Universidad Pontificia Bolivariana.

Castro. (2006). Determinación de la Eficiencia del Jacinto del Agua y Lenteja de Agua. Universidad Nacional Toribio Rodríguez Mendoza de Amazonas.

Celis, J., Junod, J., \& Sandoval, M. (2005). Recientes aplicaciones de la depuración de aguas residuales con plantas acuáticas. $14,1,17$ - 19. Chile.

Cevallos. (2015). Remediación del Agua Residual Pecuaria.

Coral, J. (2002). Tratamiento de aguas residuales domésticas mediante el cultivo de Lenteja de agua (Lemna sp.) en la cuenca del lago San Pablo. Barra, Ecuador. Universidad Técnica del Norte.

Emapacopsa. (2015). Aguas Residuales.

Fidel, M. (2012). Aguas de Cocina.

Flores. (2014). Aplicación de Humedal Artificial con Macrofitas Flotantes en la Recuperación de las ARD . San Martin.

Garcia, R. (2012). Depuración de Aguas Servidas, Utilizando Especies acuáticas.

Garcia, Z. (2012). Comparación y evaluación de tres plantas acuáticas para determinar la eficiencia de remoción de nutrientes en el tratamiento de aguas residuales domésticas. Lima, Perú: Universidad Nacional de Ingeniería. 
Haustein, A. (2004). Estudio de la Lenteja de Agua en Aguas Servidas. Investigación y monitoreo de la cuenca de los ríos Carabaya Ramis y Cabanillas en el sector Nor Oeste del Lago Titicaca . Lima, Perú: Universidad Nacional Agraria La Molina Facultad de Ciencias Forestales.

Jaramillo, M., \& Flores, E. (2012). Fitorremediación mediante el uso de dos especies vegetales Lemna minor (Lenteja de agua), y Eichornia crassipes (Jacinto de agua) en aguas residuales producto de la actividad minera. 40. Cuenca, Ecuador: Universidad Politécnica Salesiana.

Leal, G. (2006). Recolección de Especies Acuáticas.

León, M., \& Lucero, A. (2009). Estudio de Eichhornia crassipes, Lermma gibba y Azolla filiculoides en el tratamiento biológico de aguas residuales domésticas en sistemas comunitarios y unifamiliares del Cantón Cotacachi. 29-37. Ibarra, Ecuador: Universidad Técnica del Norte.

Londoño, L., \& Marín, C. (2009). Evaluación de la eficiencia de remoción de materia orgánica en humedales artificiales de flujo horizontal subsuperficial alimentados con agua residual sintética. 14- 29. Pereira, Colombia: Universidad Tecnológica de Pereira.

Lucero. (2009). Selección de Macrofitas.

Madigan. (2004). Nutrientes en las Aguas Residuales Domésticas.

Madueño, R., \& Sandoval, J. (2009). Evaluación del uso de la planta acuática Lemna para determinar la eficiencia remoción de nutrientes a escala reactor del efluente de la laguna Secundaria de la Planta CITRAR. 125. Lima, Perú: Universidad Nacional de Ingeniería. Facultad de Ingeniería Ambiental.

Metcalf, \& Inc., E. (2002). Ingeniería de aguas residuales. Tratamiento, vertido y reutilización. III. (M. Graw-Hill, Ed.) España.

MINAM. (2010). Límites Máximos Permisibles.

Morales, B. (2016). Planta de Tratamiento de Aguas Residuales.

Moreno Franco, D. (2010). Métodos para identificar, diagnosticar y evaluar el grado de eutrofia. Mexico.

Panduro, A. (2008). Sistema de descontaminación de las aguas residuales mediante el uso de plantas acuáticas, en la provincia de Coronel Portillo, Región Ucayali. . Ucayali. 
Rodríguez, C. (2001). Acción depuradora de algunas plantas acuáticas sobre las aguas residuales. 1-5. La Habana, Cuba: Instituto Superior Politécnico José A. Echevarría.

Rodríguez, N. (2009). Estudio de un biosistema integrado para el postratamiento de las aguas residuales del café utilizando macrofitas Acuáticas. 175-177. Valencia, España: Universidad Politécnica de Valencia.

Santana, M. (2010). Diagnóstico de la contaminación por aguas residuales domésticas, cuenca baja de la quebrada la Macana, San Antonio de Prado, Municipio de Medellin.

Stevenson. (1996). Factores Reguladores del Crecimiento.

Torres, J. (2007). La depuración y regeneración de las aguas mediante el Filtro de Macrófitas en Flotación. 124.

Valderrama, L. (2005). Las plantas acuáticas una alternativa para el tratamiento de aguas residuales. 3-7. Bogotá, Colombia: Unidad de Saneamiento y Biotecnología Ambiental.

Valderrama, L., Campos, C. V., \& Zapata, N. (2002). Evaluación del efecto del tratamiento con plantas acuáticas (E. crassipes, Lemna sp y L. Leavigatum) en la remoción de indicadores de contaminación fecal en aguas residuales domésticas.

Valero, M. (2006). Aplicación Tecnológica de las macrofitas a la depuración de aguas residuales con la ayuda de microrganismos. 42. Bucaramanga, Colombia: Universidad Industrial de Santander. 